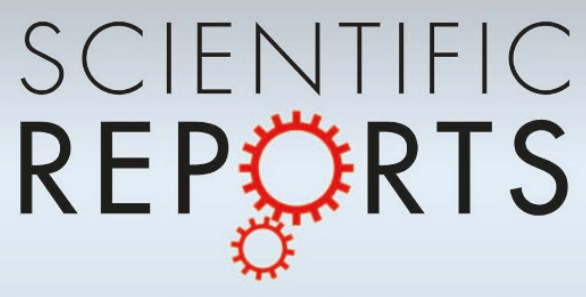

OPEN

SUBJECT AREAS:

NEUROSCIENCE

ANXIETY

DISEASES OF THE NERVOUS

SYSTEM

INNER EAR

Received

3 July 2012

Accepted

3 October 2012

Published

19 October 2012

Correspondence and requests for materials should be addressed to M.H. (mhonma@ncnp. go.jp) or K.K.

(kenichik@ncnp.go.jp)

\section{Disturbances in equilibrium function after major earthquake}

\author{
Motoyasu Honma' ${ }^{1}$ Nobutaka Endo², Yoshihisa Osada ${ }^{3}$, Yoshiharu Kim \& Kenichi Kuriyama'
}

'Department of Adult Mental Health, National Institute of Mental Health, National Center of Neurology and Psychiatry, 4-1-1 Ogawa-Higashi, Kodaira, Tokyo 187-8553, Japan, ${ }^{2}$ Department of Psychology, Kinki University, 3-4-1 Kowakae, Higashi-Osaka, Osaka 577-8502, Japan, ${ }^{3}$ Department of Psychology, Rikkyo University, 3-34-1 Nishi-lkebukuro, Toshima, Tokyo 171-8501, Japan.

Major earthquakes were followed by a large number of aftershocks and significant outbreaks of dizziness occurred over a large area. However it is unclear why major earthquake causes dizziness. We conducted an intergroup trial on equilibrium dysfunction and psychological states associated with equilibrium dysfunction in individuals exposed to repetitive aftershocks versus those who were rarely exposed. Greater equilibrium dysfunction was observed in the aftershock-exposed group under conditions without visual compensation. Equilibrium dysfunction in the aftershock-exposed group appears to have arisen from disturbance of the inner ear, as well as individual vulnerability to state anxiety enhanced by repetitive exposure to aftershocks. We indicate potential effects of autonomic stress on equilibrium function after major earthquake. Our findings may contribute to risk management of psychological and physical health after major earthquakes with aftershocks, and allow development of a new empirical approach to disaster care after such events.

ast major earthquakes have been associated with increased prevalence of psychiatric morbidities such as anxiety disorders ${ }^{1}$, mood disorders ${ }^{2}$, sleep disorders ${ }^{3,4}$, and posttraumatic stress disorder ${ }^{1,3}$ as well as neurological complaints such as dizziness ${ }^{5}$. The Tohoku earthquake on March 11 in 2011 (moment magnitude $=$ 9.0, Shindo 7 [Japanese seismic scale: see Supplementary Table 1]) and the large numbers of aftershocks (Fig. 1) was no exception (Japan Meteorological Agency, 2011: The Shindo database searching, http://www.seisvol.kishou.go.jp/eq/shindo_db/shindo_index.html); several months after the initial earthquake, significant outbreaks of dizziness occurred over a large area surrounding the epicenter of the earthquake, including in Tokyo ${ }^{6}$.

Dizziness is generally associated with neural lesions at two different levels, ranging from the distal sensory organ of equilibrium to the cortical center via the brainstem nuclei ${ }^{7}$. Ménière syndrome ${ }^{8}$, benign paroxysmal positional vertigo $(\mathrm{BPPV})^{9}$, and vestibular neuritis give rise to rotatory vertigo through organic disorders of the distal sensor of equilibrium in the inner ear ${ }^{10}$. The semicircular canal located in the inner ear sends signals to the brain via the vestibular nerve regarding the direction, speed, and acceleration of head rotation ${ }^{11}$. The semicircular canal consists of 3-dimensional tubular structures filled with endolymph containing cilia cells. When the cilia cells sense motion in the endolymph, a postural signal is transmitted to the brain. Rotatory vertigo, also called peripheral vertigo, refers to a sensation of the external world revolving around the patient or of the patient revolving gently along the circumference of a larger circle 9 . In contrast, postural vertigo, also called central vertigo, occurs due to organic disorders in the brainstem nuclei or higher cortical levels ${ }^{10}$. Postural vertigo can be described as the sensation of boarding a boat rocking in water rippling irregularly and swiftly, and often accompanies neurologic deficits such as articulation disorder or double vision?.

Both forms of vertigo are accompanied by a sensation of disequilibrium. Peripheral neurological disturbances generally enhance low-frequency and large-amplitude fluctuations of the body's center of gravity $(\mathrm{CoG})^{12}$. In contrast, central neurological disturbances generally enhance high-frequency and small-amplitude fluctuations of the $\mathrm{CoG}^{13,14}$. In addition, disequilibrium caused by unilateral peripheral neurological disturbances causes fluctuations of the CoG in the horizontal direction ${ }^{14}$, but other types cause fluctuations of the CoG in the antero-posterior direction ${ }^{12,15}$. Equilibrium dysfunction can also occur through psychological stress such as anxiety $^{16,17}$, that shows a similar pattern of CoG fluctuation to those with central neurological disturbances ${ }^{18,19}$, suggesting that anxiety may affect vestibular neural networks at higher cortical levels.

One possible explanation for the large outbreaks of dizziness after major earthquakes is that psychological stress caused equilibrium disturbance. However, there remains a plausible hypothesis that the equilibrium disturbance was caused by repetitive exposure to aftershocks; frequent physical shaking may directly disturb 

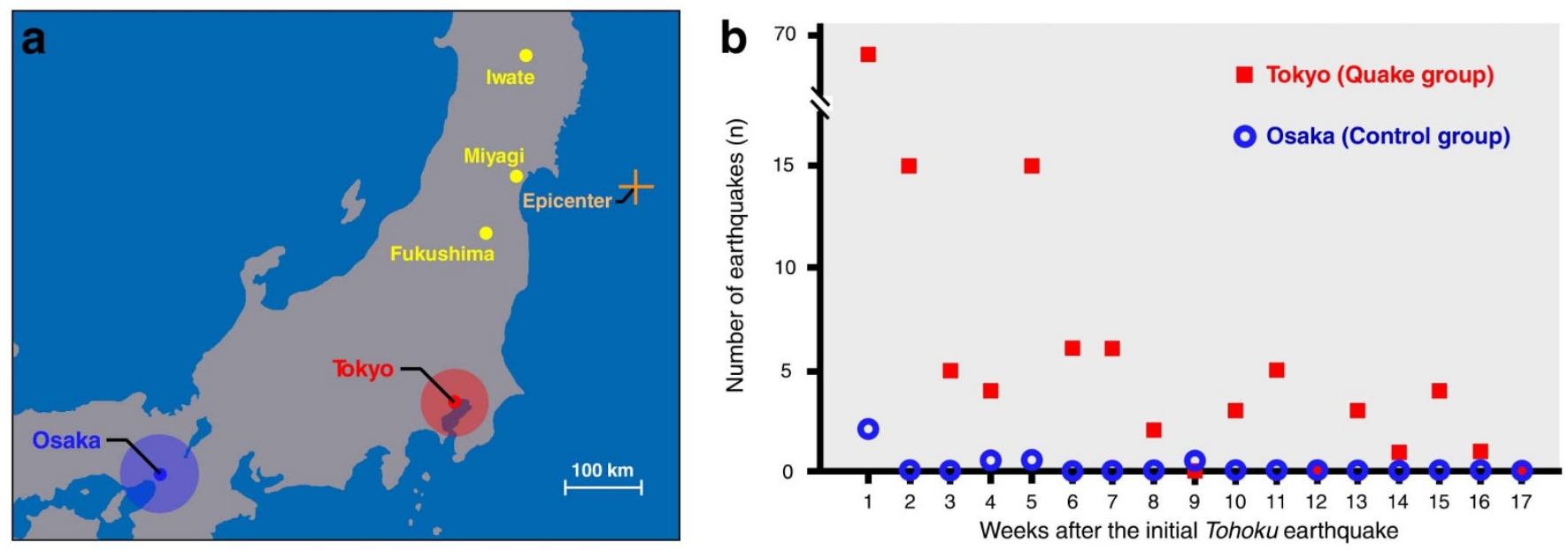

Figure $1 \mid$ Earthquakes. (a) Locations of the epicenter of the Tohoku earthquake, the sampled areas of Tokyo and Osaka, and the most affected areas of Iwate, Miyagi, and Fukushima. The distances from the epicenter to Tokyo and Osaka are approximately 350 and $700 \mathrm{~km}$, respectively. The transparent circles indicate the geographic areas within which the members of each group resided. (b) Number of aftershocks per week from March 11, 2011 to July 6, 2011 in Tokyo (Quake group) and Osaka (Control group) recorded more than Shindo level 2.

the functioning of the semicircular canal system. A preliminary experimental trial suggested that equilibrium functions potentially exhibit habituation and adaptation to novel physical surroundings ${ }^{20}$. Thus, to examine these potential associations with equilibrium disturbance, we conducted an intergroup trial on postural sway in individuals repeatedly exposed to aftershocks versus rarely exposed individuals. Furthermore, we examined the psychological effects of stress such as anxiety and depression on equilibrium disturbance by conducting within-group correlation analyses and power spectrum analyses of postural sway.

\section{Results}

Equilibrium dysfunction in the Quake group. We recruited 101 individuals who either resided in Tokyo prefecture and were exposed to a large number of aftershocks over a four-month period (Quake group: $n=52$ ) or resided in Osaka prefecture and were rarely exposed to aftershocks (Control group: $n=49$ ), on the grounds for radiation ${ }^{21}$ and social ${ }^{22}$ stresses. We measured several stabilometric parameters of balance (total path length [TPL], rectangular area [REC-Area], and enveloped area [ENV-Area]), calculated by detecting the CoG continuously on a force platform. The standard test battery was conducted for $60 \mathrm{sec}$ under both eyes open (EO) and eyes closed (EC) conditions under equivalent environments for both the Quake and Control groups from July 6-15, 2011, approximately 17 weeks after the Tohoku earthquake. We simultaneously administered several clinical questionnaires including the State-Trait Anxiety Inventory (STAI-state and -trait) ${ }^{23}$, the Beck Depression Inventory $(\mathrm{BDI})^{24}$, the Impact of Event Scale Revised (IES-R) ${ }^{25}$, and the Pittsburgh Sleep Quality Index (PSQI) ${ }^{26}$ as markers of psychological stress. We also administered additional questionnaires concerning specific damages (injuries, lost property, or deaths of family members) caused by the Tohoku earthquake, complaints of dizziness and headache, and degree of anxiety over radiation and earthquakes.

Between-group differences are shown in Table 1. None of the participants in the study were injured, lost property, or lost family members as a direct result of the earthquake, and none of the participants complained of any neurological symptoms, including dizziness. Unpaired two-tailed $t$ tests revealed no significant differences in anxiety over radiation $(p>0.20)$ or earthquakes $(p>$ 0.10 ) between the groups.

There were significant differences in each of the stabilometric parameters under the EC condition between the groups (TPL: $t_{99}$
$=3.091, p=0.003$; REC-Area: $t_{99}=2.759, p=0.007$; ENV-Area: $t_{99}$ $=3.016, p=0.003$ ), but not under the EO condition (all: $p>0.10$ ). There were no significant differences between the groups in the clinical assessment inventories (all: $p>0.40$ ). Taken together, a greater but symptom-limited disturbance of the equilibrium function in the Quake group was observed compared to the Control group.

Psychological influences on equilibrium function. There were no significant correlations between the clinical questionnaires and the stabilometric parameters under either the EO or EC condition in the Control group (Pearson's correlation coefficient, all: $p>$ $0.10)$. However, there were significant correlations between each stabilometric parameter and STAI-state (TPL: $r=0.502, p<$ 0.0001; REC-Area: $r=0.494, p<0.0001$; ENV-Area: $r=0.495, p$ $<0.0001$; Fig. 2a-c), STAI-trait (TPL: $r=0.420, p=0.002$; RECArea: $r=0.337, p=0.008$; ENV-Area: $r=0.352, p=0.011$; Fig. $2 \mathrm{~d}$ f), and BDI (TPL: $r=0.364, p=0.007$; REC-Area: $r=0.336, p=$ 0.015 ; ENV-Area: $r=0.352, p=0.010$; Fig. $2 \mathrm{~g}-\mathrm{i}$ ) under the EC condition in the Quake group (Fig. 2a-i), but not under the EO condition (all: $p>0.10$ ). There were no significant correlations between other factors (body-mass index, athletic history, anxiety over radiation and earthquakes, and subjective sense of sway,) and any of the stabilometric parameters (all: $p>0.10$ ).

To verify the additional hypothesis that psychological stress may affect equilibrium function differently according to the intensity of the stress, we conducted the above statistical analyses for both higher- and the lower-stress Quake subgroups, as determined by the median values of each clinical assessment inventory. The STAI-state (median value $=46)$ was correlated with the TPL $(r=$ $0.495, p=0.007$; Fig. 3a), REC-Area ( $r=0.431, p=0.022$; Fig. 3b), and ENV-Area $(r=0.514, p=0.005$; Fig. $3 c)$ in the higher STAIstate group $(n=28)$, although it was not correlated with any of the stabilometric measurements (all: $p>0.10$; Fig. 3a-c) in the lower STAI-state group $(n=24)$. The STAI-trait (median value $=50)$ was correlated with the TPL $(r=0.436, p=0.021$; Fig. 3d), but was not correlated with REC-Area $(p>0.10$; Fig. 3e) or ENV-Area $(P>0.05$; Fig. 3f) in the higher STAI-trait group $(n=28)$. It also was not correlated with any of the stabilometric measurements (all: $p>$ 0.05; Fig. 3d-f) in the lower STAI-trait group $(n=24)$. The BDI (median value $=26$ ) was not correlated with any of the stabilometric measurements in either the higher $(n=27$, all: $p>0.05$; Fig. $3 g-i)$ or lower $(n=25$, all: $p>0.10$; Fig. $3 g-i)$ BDI groups. These results indicate that individuals with greater anxiety symptoms in the Quake 
Table 1 | Comparison of Quake with Control groups

$\frac{\text { Quake }(n=52)}{\text { Average (SEM) }} \quad \frac{\text { Control }(n=49)}{\text { Average (SEM) }}$

$t$

p

Demographic information

Sex

Female (n) 32

Male (n)

Age

32

Body-mass index $\left(\mathrm{kg} / \mathrm{m}^{2}\right)$

$20.88(0.11)$

Athletic history (year)

Primary victim in the earthquake (n)

Individual with dizziness (n)

Psychological distress against disaster

Anxiety over radiation

Anxiety over earthquake

$20.27(0.30)$

$7.90(0.71)$

0

0

Clinical questionnaires

STAl-state

STAl-trait

BDI

IES-R

PSQI

46.17 (3.87)

59.37 (3.39)

19

$20.12(0.05)$

$20.59(0.32)$

$7.02(0.75)$

0

0

$43.85(1.54)$

$47.91(1.38)$

$10.82(0.91)$

$25.76(2.28)$

$3.25(0.27)$

40.02 (3.19)

$51.90(3.82)$

1.460

1.212

$-0.735$

0.857

0.228

Stabilometric measurements

TPL

Eyes open $(\mathrm{mm})$

Eyes closed $(\mathrm{mm})$

$428.7(16.2)$

599.9 (24.3)

$43.80(1.11)$

$48.14(1.34)$

$10.20(0.94)$

$24.78(1.87)$

$3.61(0.34)$

-

0.393

REC-Area

Eyes open $\left(\mathrm{mm}^{2}\right)$

Eyes closed $\left(\mathrm{mm}^{2}\right)$

$521.0(73.9)$

$675.9(58.4)$

$412.2(26.6)$

495.8 (23.2)

0.026

$-0.474$

0.475

0.131

$-0.841$

-

ENV-Area

Eyes open $\left(\mathrm{mm}^{2}\right)$

Eyes closed $\left(\mathrm{mm}^{2}\right)$

$156.4(15.3)$

402.3 (49.7)

$467.9(46.8)$

3.091

0.003

$217.2(19.6)$

$137.7(17.2)$

1.314

2.759

0.191

144.3 (13.6)

0.813

$\mathbf{0 . 0 0 7}$

Pearson's $X^{2}$ test showed no significant difference in ratio of sex among groups $\left(X^{2}=0.001, p=0\right.$. were measured using a visual analogue scale. Results with $p$ values $<0.05$ are shown in bold.

group showed a marked relationship between the severity of anxiety and equilibrium dysfunction. Greater anxiety, as reflected by STAIstate scores, was associated with worse equilibrium function in the Quake group.

Anxiety and inner ear dysfunction. To determine the origin of the equilibrium dysfunction associated with repetitive exposure to aftershocks, we conducted a power spectrum analysis on the CoG using fast Fourier transform. The power was calculated for lowfrequency bands $(0.0-0.1 \mathrm{~Hz})$ and high-frequency bands (1.0$10 \mathrm{~Hz}$ ) as percentages of the total. We compared the power spectra of the groups using unpaired $t$ tests and calculated correlation coefficients between the power spectra and the clinical assessment inventories. The low-frequency power of the CoG fluctuation in the antero-posterior direction in the Quake group was significantly greater than that in the Control group $\left(t_{99}=3.01, p=0.003\right.$, Fig. 4a) under the EC condition. In contrast, the high-frequency power of the CoG fluctuation in the antero-posterior direction in the Quake group was significantly lower than that of the Control group $\left(t_{99}=-2.429, p=0.017\right.$; Fig. $\left.4 \mathrm{~b}\right)$. The low-frequency power of the CoG fluctuation was positively correlated with STAI-state $(r=$ $0.315, p=0.023$; Fig. $4 \mathrm{c})$, and the high-frequency power of the CoG fluctuation was negatively correlated with STAI-state $(r=-0.401, p$ $=0.003$; Fig. $4 \mathrm{~d}$ ) in the Quake group. However, neither frequency power was correlated with STAI-state, -trait, or BDI in the Control group (all: $p>0.10$ ).

\section{Discussion}

Although there were no complaints of dizziness and no significant differences in subjective psychological stress between the residents of Tokyo and Osaka prefectures, more severe equilibrium dysfunction was detected in the Quake group, indicating that repetitive exposure to aftershocks can evoke equilibrium dysfunction 4 months after a major earthquake.

The low- and high-frequency band powers for the CoG in the antero-posterior direction were positively and negatively correlated, respectively, with the current anxiety state as reflected by STAI-state scores in the Quake group. Specifically, positive correlations between all stabilometric parameters and the STAI-state score were observed under the EC condition in the Quake group. Thus, equilibrium dysfunction in the Quake group may also have been affected by psychological stress due to repetitive exposure to aftershocks. It has been suggested that vestibular-corticolimbic interaction occurs via two separate neural pathways at the higher cortical and the medullary levels ${ }^{19}$. Anxiety may enhance equilibrium dysfunction both in healthy subjects ${ }^{15}$ and in patients with vestibular dysfunctions such as Ménière disease ${ }^{27}$ and $\mathrm{BPPV}^{28}$, in which an increase in the highfrequency band power for CoG has been found, suggesting that vestibular-corticolimbic interaction could be promoted by the neural pathway at the higher cortical level. However, equilibrium dysfunction exhibiting the increase in the low-frequency band power of the CoG in the Quake group, likely caused by bilateral inner ear disturbance due to repetitive physical shaking, may have been modulated by vestibular-corticolimbic interaction through the other neural pathway at the medullary level.

The parabrachial nuclei receive input from the vestibular nuclei as well as the insular, amygdala, and bed nuclei of the stria terminalis, which are involved in emotional processing ${ }^{18}$. In particular, the bed nuclei of the stria terminalis are involved in longer-lasting emotional responses such as anxiety ${ }^{29,30}$. Anxiety is typically integrated with vestibular information in the corticolimbic area ${ }^{31}$; however, it may also be translated by autonomic neurotransmission. Somatic senses such as startle stimuli often form an autonomic reflex via association with psychological stress, called a startle response ${ }^{32}$. Physical shaking 

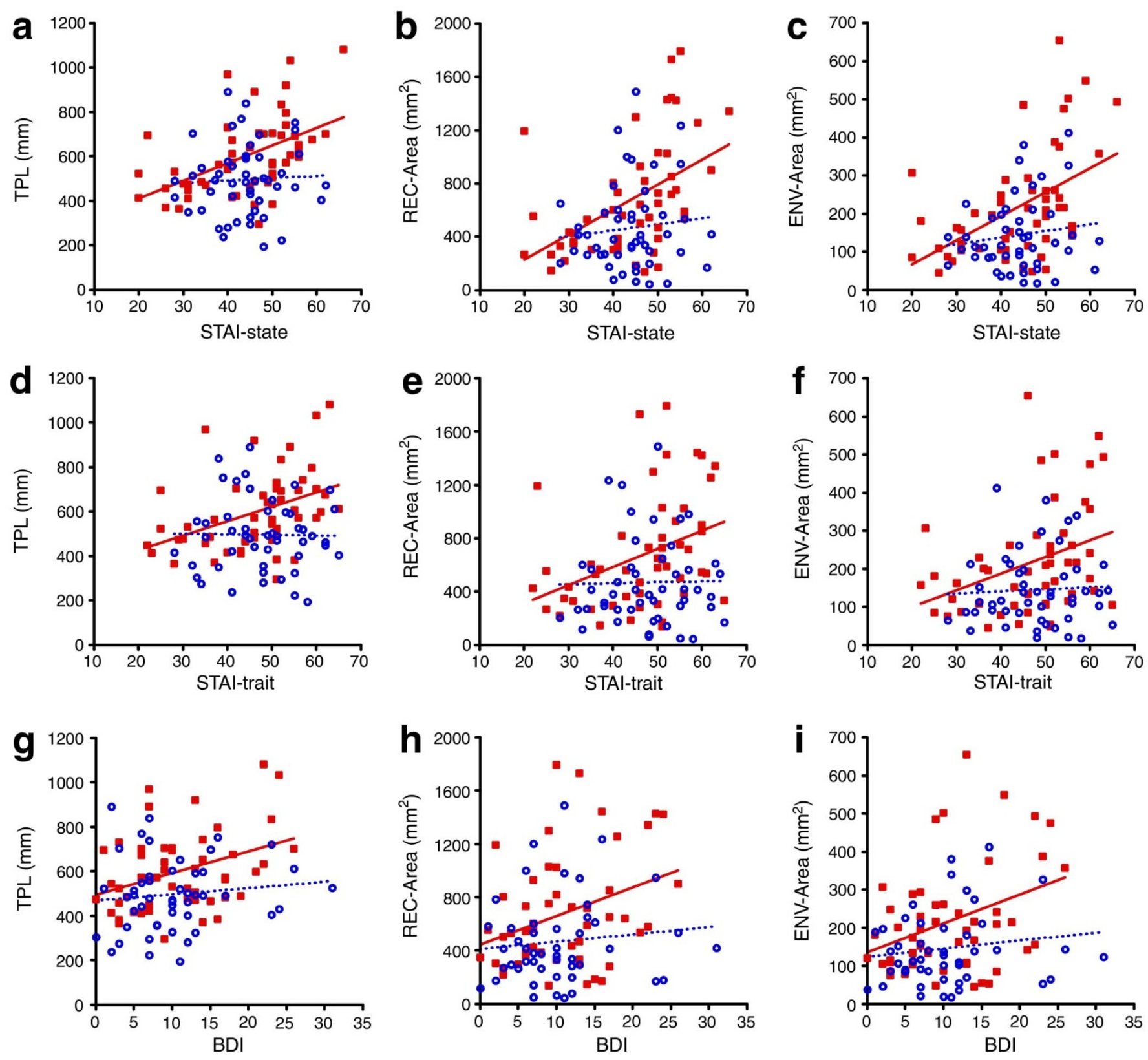

Figure $2 \mid$ Psychological distress affects equilibrium dysfunction. TPL, REC-Area, and ENV-Area were significantly correlated with STAI-state, -trait, and BDI only under the EC condition in the Quake group. The STAI-state was strongly correlated with TPL (a), REC-Area (b), and ENV-Area (c). The STAI-trait also was strongly correlated with TPL (d), REC-Area (e), and ENV-Area (f). The BDI was correlated with TPL (g), REC-Area (h), and ENV-Area (i). None of the stabilometric measurements in the Control group were significantly correlated with STAI-state, -trait, or BDI. The red squares with solid regression lines represent the Quake group, and the blue circles with dashed regression lines represent the Control group.

may be associated with autonomic regulation; thus, contextual conditioning may have been formed by repetitive aftershocks experienced by the Quake group individuals. Animal neuroanatomical findings indicate that the vestibulo-parabrachial pathway, which reciprocally integrates not only vestibular and corticolimbic but also autonomic information, is present in the brain stem ${ }^{19}$. Even considering that the autonomic center is involved in the reticular formation of the medulla, humans may also integrate autonomic, vestibular, and corticolimbic information at the medullary level, potentially enhancing inner ear disturbance.

Visual information is also combined with vestibular information at both the higher cortical and brain stem levels ${ }^{33}$ at the superior colliculi of the mesencephalon and the tegmentum of the pons, in which secondary vestibular neurons make synaptic connections with the oculomotor nuclei and the abducens nuclei, respectively ${ }^{34}$. The medial and lateral rectus muscles, which are innervated by the somatic motor nerves of the oculomotor and the abducens, respectively, rotate the eyes when the head turns ${ }^{35}$. This visual compensation supporting equilibrium function may arise from an oculovestibular reflex together with a possible neural connection associated with visuo-vestibular interactions at the higher cortical level ${ }^{36}$. Therefore, equilibrium dysfunction is amplified when the eyes are closed.

The current study has several potential limitations. First, our results may demonstrate regional differences in the effects of aftershocks on equilibrium function; however, the cross-sectional study protocol did not exclude individual differences in evaluating the specific impacts of this major earthquake on equilibrium function. 

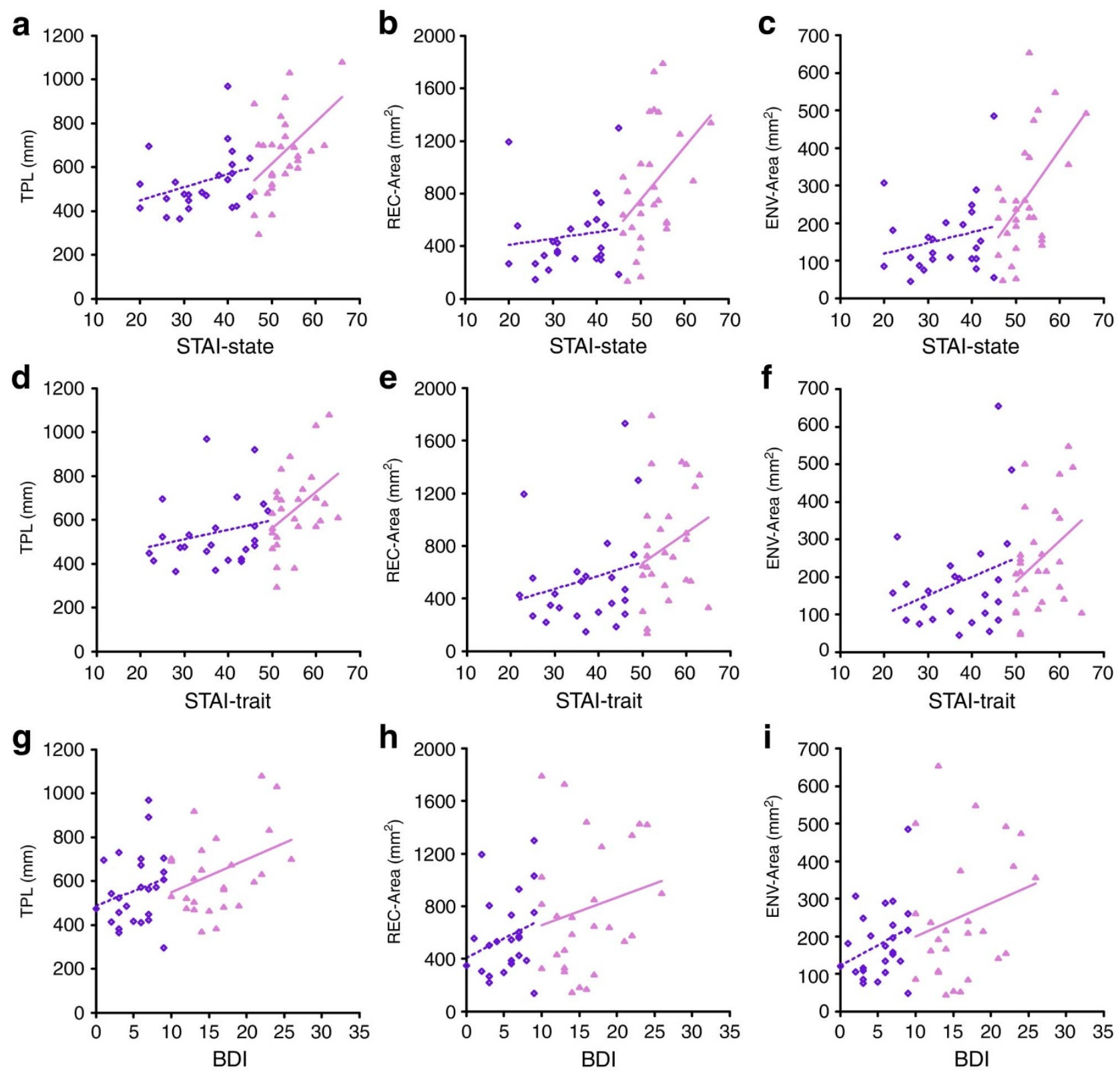

Figure $3 \mid$ Differences between higher- and lower-stress members of the Quake group in the relationships between psychological stress and equilibrium dysfunction under the EC condition. STAI-state was significantly correlated with TPL (a), REC-Area (b), and ENV-Area (c) in higher scoring individuals; however, it was not correlated with TPL (a), REC-Area (b), or ENV-Area (c) in lower scoring individuals. STAI-trait was significantly correlated with TPL (d), but was not correlated with REC-Area (e) or ENV-Area (f) in higher scoring individuals. In the lower scoring subgroup, STAItrait was not correlated with any stabilometric measurement (d, TPL; e, REC-Area; f, ENV-Area). The BDI was not correlated with any stabilometric measurement for either higher scoring individuals (g, TPL; h, REC-Area; i, ENV-Area) or lower scoring individuals (g, TPL; h, REC-Area; i, ENV-Area). The purple diamonds with dashed regression lines represent the lower group, and the pink triangles with solid regression lines represent the higher group.

Second, we were not able to examine direct relationships between the clinical symptoms of dizziness and equilibrium dysfunction. To investigate the effects of the earthquake on equilibrium function, we excluded patients with mental disorders, including those who had experienced dizziness and/or clinical levels of anxiety, because dizziness patients may simultaneously have several anxiety symptoms as one of the common sequelae of dizziness ${ }^{8}$. For similar reasons, subjects were included only if their anxiety and depression symptoms did not reach threshold levels for the respective screening criteria. Third, because the airborne radiation doses in both regions were not noticeably different between the period from March 11 to July 6 in 2011 (Tokyo: average, $0.071 \mu \mathrm{Sv} / \mathrm{h}$; Osaka: $0.043 \mu \mathrm{Sv} / \mathrm{h}$ ) and previously (Tokyo: 0.028-0.079 $\mu \mathrm{Sv} / \mathrm{h}$; Osaka: 0.042-0.061 $\mu \mathrm{Sv} / \mathrm{h}$ )
(Ministry of Education, Culture, Sports, Science \& Technology in Japan, 2011: The database of monitoring information of environmental radioactivity level, http://radioactivity.mext.go.jp/ja/monitoring_by_prefecture/), individual acute doses were not estimated in the current study. Thus, there is room for further research into the effects of radiation exposure on equilibrium function. In addition, although equilibrium function could be influenced by daily activities and environmental factors such as commuter traffic and fitness habits, it was difficult to control these lifestyle variables. We were able to recruit participants from groups that had broadly similar living environments. Finally, we did not observe significant differences in psychological effects from the earthquake as measured by the STAI and BDI. However, it is uncertain whether these inventories 

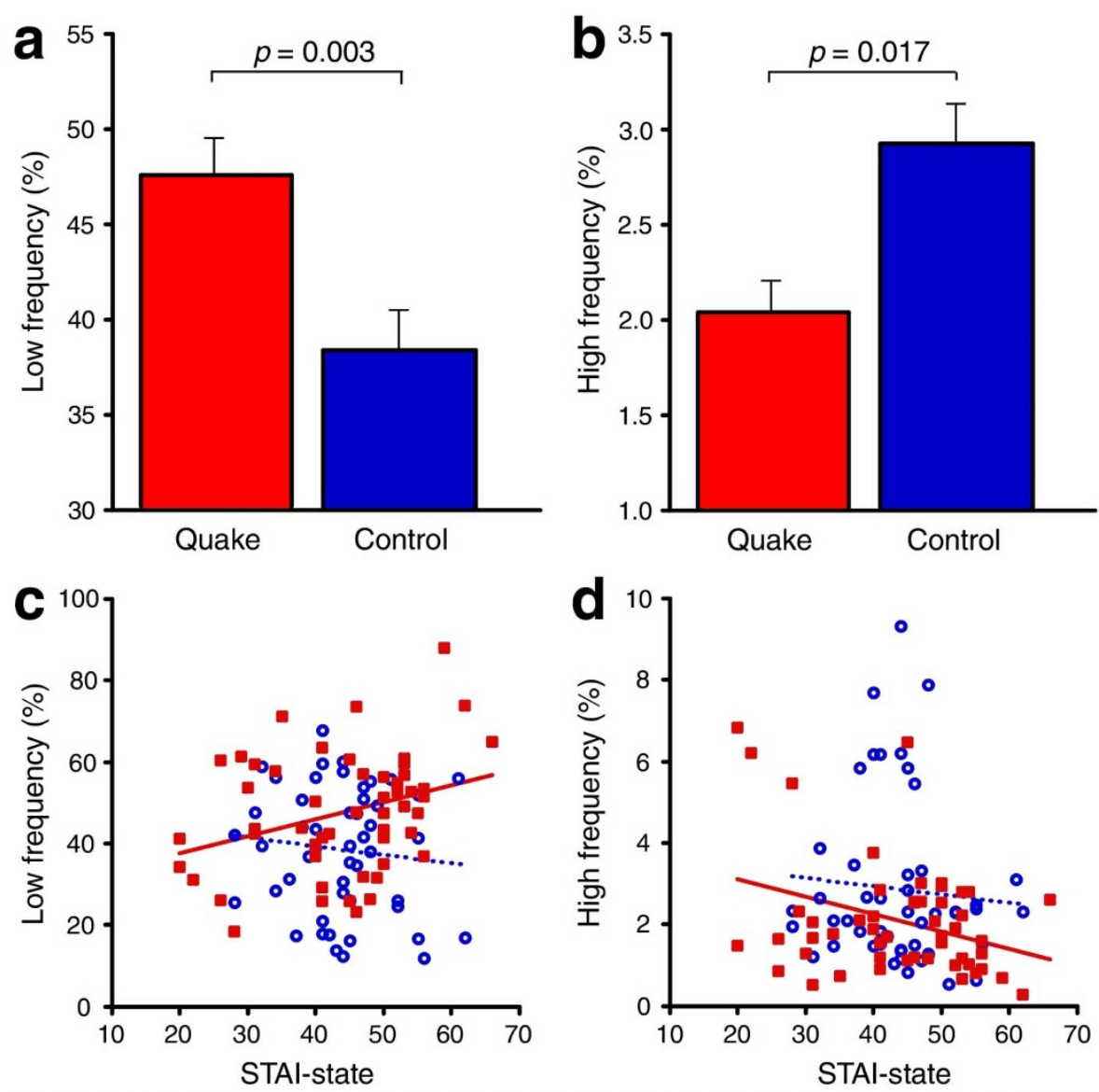

Figure $4 \mid$ Anxiety is associated with peripheral vestibular dysfunction. The power spectra for CoG fluctuations were divided into low- and highfrequency bands along the antero-posterior axis under the EC condition. (a) The percent CoG fluctuation in the low-frequency band in the Quake group was significantly greater than that in the Control group. (b) The percent CoG fluctuation in the high-frequency band in the Quake group was significantly lower than that in the Control group. (c) The percent CoG fluctuation in the low-frequency band was positively correlated with STAI-state. (d) The percent CoG fluctuation in the high-frequency band was negatively correlated with STAI-state. The power spectra for CoG fluctuations in both the lowand high-frequency bands for the Control group were not correlated with STAI-state. The red bars and red squares with solid regression lines represent the Quake group, and the blue bars and blue circles with dashed regression lines represent the Control group. Error bars indicate SEM.

are able to specifically evaluate post-earthquake effects. Further research on the nature of post-disaster psychological suffering would be valuable.

We have provided evidence that repetitive exposure to earthquakes causes dysfunction in the inner ear. In addition, the current study indicates potential effects of autonomic stress on equilibrium function. Equilibrium dysfunction caused by earthquakes may be affected by physical invasion and automatic stress, as well as interactions between them, through vestibular neural integration. The present results contribute to risk management of mental and physical health after major earthquakes with aftershocks, and may allow development of a new empirical approach to disaster care during and after such events. Medical treatment for equilibrium dysfunction such as a rehabilitation of balance control ${ }^{37}$ and pharmacological treatment for vestibular dysfunction ${ }^{38}$ could alleviate psychological distress, including anxiety symptoms of earthquake victims, without direct psychological support. Disaster victims often prefer not to discuss post-disaster trauma, but may be willing to seek treatment for their physical symptoms. Medical approaches to equilibrium dysfunction may be helpful for earthquake disaster victims, although further trials are needed.

\section{Methods}

Participants. We recruited 101 university students who had no history of drug or alcohol abuse or neurological, ophthalmological, or psychiatric disorders. The 52 members of the Quake group were exposed to a large number of aftershocks over a four-month period and the 49 members of the Control group were rarely exposed to aftershocks were attending private universities in Tokyo and Osaka, respectively, which have similar living environments. The members of each group had a similar average commuting distance to school (Quake: mean $15.5 \pm 1.2 \mathrm{~km}$; Control: mean $16.8 \pm 1.8 \mathrm{~km}$ ). These similarities provided a degree of control for two possible influences, radioactive exposure from the devastated Fukushima nuclear power station $^{21}$ and an urban lifestyle that affects social stress processing ${ }^{22}$. We conducted the examinations on July 6-15, 2011 approximately 17 weeks after the Tohoku earthquake. Based on a survey, all participants had resided within their local area since the earthquake on March 11,2011. During the examinations, no aftershocks were recorded by the Shindo database. The experimental procedure was in accordance with the Declaration of Helsinki and was approved by the local Research Ethics Committees in Kinki University and Rikkyo University. Written informed consent was obtained from all participants prior to the study.

Experimental procedures. The experimental conditions in the laboratories for both groups were equalized with respect to the slope angle of the floor $\left(<0.05^{\circ}\right.$ in the rightleft and antero-posterior directions), noise level $(<20 \mathrm{~dB})$, temperature (average: 25.2 degree, SEM: 0.18), humidity (42.4\%, 0.64), and illuminance (407 lux, 8.8). Stabilometer was performed using a computerized force platform according to a standard protocol for clinical examination of equilibrium function (UM-BAR2; HuTech Co., Ltd, Tokyo, Japan). The stabilometer is equipped with two vertical force transducers to determine instantaneous fluctuations in the CoG, and samples fluctuations in the CoG at a frequency of $60 \mathrm{~Hz}$. Subjects stood erect on the stabilometer with feet together while gazing at a fixation point $150 \mathrm{~cm}$ distant in a horizontal direction from the subject's eyes. All subjects were tested in three trials for $60 \mathrm{sec}$ each under eyes open (EO) and eyes closed (EC) conditions alternately and successively. The postural sway was represented as an $x$ (right-left) $-y$ (anteroposterior) curve in the total path length (TPL), rectangular area (REC-Area), and enveloped area (ENV-Area) of the CoG. TPL represents the amount of CoG displacement per unit time. TPL was calculated using equation 1. ENV-Area, which is 
1995 Hanshin-Awaji earthquake in Japan. Acta Psychiatr Scand 93, 477-481 (1996).

4. Varela, E., Koustouki, V., Davos, C. H. \& Eleni, K. Psychological consequences among adults following the 1999 earthquake in Athens, Greece. Disasters 32, 280-291 (2008)

5. Chen, C. H. et al. Long-term psychological outcome of 1999 Taiwan earthquake survivors: a survey of a high-risk sample with property damage. Compr Psychiatry 48, 269-275 (2007)

6. Pollack, A. \& Bradsher, K. In Japan, aftershocks are also felt from within. The New York Times www.nytimes.com/2011/04/14/world/asia/14quake.html (2011).

7. Wackym, P. A., Hannley, M. T., Runge-Samuelson, C. L., Jensen, J. \& Zhu, Y. R. Gamma Knife surgery of vestibular schwannomas: longitudinal changes in vestibular function and measurement of the Dizziness Handicap Inventory. J Neurosurg 109, 137-143 (2008).

8. Minor, L. B., Schessel, D. A. \& Carey, J. P. Ménière's disease. Curr Opin Neurol 17, 9-16 (2004).

9. Fetter, M. Assessing vestibular function: which tests, when? J Neurol 247, 335-342 (2000).

$$
\mathrm{ENV}-\text { Area }=\sum_{i=1}^{120} \mathrm{R}_{i} \cdot \mathrm{R}_{i+1} \cdot \sin \theta / 2
$$

$$
\mathrm{REC}-\text { Area }=\left(\mathrm{X}_{\max }-\mathrm{X}_{\min }\right) \cdot\left(\mathrm{Y}_{\max }-\mathrm{Y}_{\min }\right)
$$

We defined the low- and high-frequency bands for the CoG in both the horizontal and antero-posterior direction as $0-0.1 \mathrm{~Hz}$ and $1.0-10.0 \mathrm{~Hz}$, respectively, based on previously reported protocols ${ }^{12-15}$, even for those bands that varied among low- $(<$ $0.05-1.0 \mathrm{~Hz})$ and high-frequency $(>1.0-3.0 \mathrm{~Hz})$ band power windows. The power spectrum was calculated as a percentage of the total.

\section{Clinical assessments. We simultaneously administered several clinical} questionnaires including the State-Trait Anxiety Inventory (STAI-state and -trait) ${ }^{23}$ the Beck Depression Inventory (BDI) ${ }^{24}$, the Impact of Event Scale Revised (IES-R) ${ }^{25}$, and the Pittsburgh Sleep Quality Index (PSQI) ${ }^{26}$ as markers of psychological stress. The STAI separately measures state anxiety and trait anxiety. The STAI separately measures state anxiety, which reflects the transitory emotional state or condition of the subject characterized by subjective, consciously perceived feelings of tension and apprehension, and trait anxiety, which reflects relatively stable individual differences in anxiety proneness and refers to a general tendency to respond with anxiety to perceived threats in the environment. Both the state and trait sections of this questionnaire comprise 20 questions, with total scores on each section ranging from 20 to 80 . STAI-state scores of $\geq 51$ and STAI-trait scores of $\geq 55$ suggest diagnoses of anxiety disorder and pathological vulnerability to threat as a personality trait. The BDI is a series of questions developed to measure the intensity, severity, and depth of depression in patients with psychiatric diagnoses. It consists of 21 items measuring symptoms of depression, such as pessimism, sense of failure, guilt, self-dislike, suicidal ideas, insomnia, and weight loss. The total score is calculated as the sum of all items and ranges from 0 to 63 (0-10: normal, 11-16: mild mood disturbance, 17-20: borderline clinical depression, 20-30: moderate depression, 31-40: severe depression, $>40$ : extreme depression). The IES-R has 22 questions, 5 of which were added to the original Horowitz IES to heighten the concordance rate for diagnosis of posttraumatic stress disorder (PTSD). The tool is an appropriate instrument for measuring the subjective response to a specific traumatic event in adults, particularly for the response sets of intrusion, avoidance, and hyperarousal. The total score ranges from 0 to 88 (score $\geq 24$ : partial PTSD or at least some of the symptoms, score 33: the best cut-off score for a probable diagnosis of PTSD). The PSQI is a self-rated questionnaire that assesses sleep quality and disturbance over a 1-month interval. The PSQI consists of seven components: subjective sleep quality, sleep latency, sleep duration, habitual sleep efficiency, sleep disturbance, use of sleep medication, and daytime dysfunction. The sum of the scores for these seven components ranges from 0 to 21 . A total score of $\geq 5$ indicates pathologically poor sleep. We also administered additional questionnaires concerning specific damages (injuries, lost property, or deaths of family members) caused by the Tohoku earthquake, complaints of dizziness and headache. The add-on questionnaires concerning specific damages from the Tohoku earthquake, the degree of anxiety over radiation and earthquakes were measured using a visual analogue scale. The higher scores were associated with more intense feelings for each state.

Statistical analysis. Unpaired $t$ test was used to examine the differences between Quake and Control groups. The relationships between the clinical questionnaires and stabilometric parameters were analyzed by Pearson's correlation coefficient. All tests were two tailed. Results are presented as the mean and standard error of the mean (SEM). A $p$ value of 0.05 was considered statistically significant.

1. Ehring, T., Razik, S. \& Emmelkamp, P. M. Prevalence and predictors of posttraumatic stress disorder, anxiety, depression, and burnout in Pakistani earthquake recovery workers. Psychiatry Res 185, 161-166 (2011).

2. Anwar, J., Mpofu, E., Matthews, L. R., Shadoul, A. F. \& Brock, K. E. Reproductive health and access to healthcare facilities: risk factors for depression and anxiety in women with an earthquake experience. BMC Public Health 11, 523 (2011).

3. Kato, H., Asukai, N., Miyake, Y., Minakawa, K. \& Nishiyama A. Post-traumatic symptoms among younger and elderly evacuees in the early stages following the
10. Bronstein, A. M. Vision and vertigo: some visual aspects of vestibular disorders. J. Neurol. 251, 381-387 (2004).

11. Zupan, L. H., Peterka, R. J. \& Merfeld, D. M. Neural processing of gravito-inertial cues in humans. I. Influence of the semicircular canals following post-rotatory tilt. J Neurophysiol 84, 2001-2015 (2000).

12. Goto, F., Kabeya, M., Kushiro, K., Ttsutsumi, T. \& Hayashi, K. Effect of anxiety on antero-posterior postural stability in patients with dizziness. Neurosci Lett 487, 204-206 (2011)

13. Yoneda, S. \& Tokumasu, K. Frequency analysis of body sway in the upright posture. Statistical study in cases of peripheral vestibular disease. Acta Otolaryngol 102, 87-92 (1986).

14. Kapoula, Z. et al. Medio-lateral postural instability in subjects with tinnitus. Front Neurol 2, 35 (2011).

15. Wada, M., Sunaga, N. \& Nagai, M. Anxiety affects the postural sway of the anteroposterior axis in college students. Neurosci Lett 302, 157-159 (2001).

16. Redfern, M. S., Furman, J. M. \& Jacob, R. G. Visually induced postural sway in anxiety disorders. J Anxiety Disord 21, 704-716 (2007).

17. Jacob, R. G., Redfern, M. S. \& Furman, J. M. Space and motion discomfort and abnormal balance control in patients with anxiety disorders. J Neurol Neurosurg Psychiatry 80, 74-78 (2009).

18. Balaban, C. D. Projections from the parabrachial nucleus to the vestibular nuclei: potential substrates for autonomic and limbic influences on vestibular responses. Brain Res 996, 126-137 (2004).

19. Balaban, C. D., Jacob, R. G. \& Furman, J. M. Neurologic bases for comorbidity of balance disorders, anxiety disorders and migraine: neurotherapeutic implications. Expert Rev Neurother 11, 379-394 (2011).

20. Oude Nijhuis, L. B., Allum, J. H., Valls-Solé, J., Overeem, S. \& Bloem, B. R. First trial postural reactions to unexpected balance disturbances: a comparison with the acoustic startle reaction. J Neurophysiol 104, 2704-2712 (2010).

21. Christodouleas, J. P. et al. Short-term and long-term health risks of nuclearpower-plant accidents. N Engl J Med 364, 2334-2341 (2011).

22. Lederbogen, F. et al. City living and urban upbringing affect neural social stress processing in humans. Nature 474, 498-501 (2011).

23. Spielberger, C. D., Gorsuch, R. L. \& Lushene, R. E. in Manual for the State-Trait Anxiety Inventory (Consulting Psychologists Press, 1970).

24. Beck, A. T. \& Steer, R. A. Internal consistencies of the original and revised Beck Depression Inventory. J Clin Psychol 40, 1365-1367 (1984).

25. Weiss, D. S. \& Marmar, C. R. in Assessing Psychological Trauma and PTSD (ed. Wilson, P. J. \& Keane, M. T. ) 399-411 (Guilford Press, 1996).

26. Buysse, D. J., Reynolds, C. F., Monk, T. H., Berman, S. R. \& Kupfer, D. J. The Pittsburgh Sleep Quality Index: a new instrument for psychiatric practice and research. Psychiatry Res 28, 193-213 (1989).

27. Havia, M., Kentala, E. \& Pyykkö, I. Postural instability in Meniere's disease $J$ Vestib Res 14, 37-46 (2004).

28. Stambolieva, K. \& Angov, G. Postural stability in patients with different durations of benign paroxysmal positional vertigo. Eur Arch Otorhinolaryngol 263, 118-122 (2006).

29. Walker, D. L., Toufexis, D. J. \& Davis, M. Role of the bed nucleus of the stria terminalis versus the amygdala in fear, stress, and anxiety. Eur J Pharmacol 463, 199-216 (2003)

30. Sink, K. S., Walker, D. L., Yang, Y. \& Davis, M. Calcitonin gene-related peptide in the bed nucleus of the stria terminalis produces an anxiety-like pattern of behavior and increases neural activation in anxiety-related structures. J Neurosci 31, 1802-1810 (2011).

31. Kalueff, A. V. et al. The regular and light-dark Suok tests of anxiety and sensorimotor integration: utility for behavioral characterization in laboratory rodents. Nat Protoc 3, 129-136 (2008).

32. Parker, K. J. et al. Somatic and neuroendocrine responses to standard and biologically salient acoustic startle stimuli in monkeys. Psychoneuroendocrinology 36, 547-556 (2011).

33. Herman, R., Mixon, J., Fisher, A., Maulucci, R. \& Stuyck, J. Idiopathic scoliosis and the central nervous system: a motor control problem. The Harrington lecture, 1983. Scoliosis Research Society. Spine 10, 1-14 (1985). 
34. Künzle, H. The cerebellar and vestibular nuclear complexes in the turtle. I. Projections to mesencephalon, rhombencephalon, and spinal cord. J Comp Neurol 242, 102-121 (1985).

35. Grantyn, A., Ong-Meang Jacques, V. \& Berthoz, A. Reticulo-spinal neurons participating in the control of synergic eye and head movements during orienting in the cat. II. Morphological properties as revealed by intra-axonal injections of horseradish peroxidase. Exp Brain Res 66, 355-377 (1987).

36. Redfern, M. S., Yardley, L. \& Bronstein, A. M. Visual influences on balance. J Anxiety Disord 15, 81-94 (2001).

37. Orrell, A. J., Eves, F. F. \& Masters, R. S. Motor learning of a dynamic balancing task after stroke: implicit implications for stroke rehabilitation. Phys Ther 86, 369-380 (2006).

38. Pompeiano, O., Manzoni, D., D'Ascanio, P. \& Andre, P. Noradrenergic agents in the cerebellar vermis affect adaptation of the vestibulospinal reflex gain. Brain Res Bull 35, 433-444 (1994).

\section{Acknowledgements}

This work was partially supported by a Japanese Psychological Association grant to support recovery from the Great East Japan Earthquake Disaster (to M.H.) and by a grant from the
Core Research for Evolutional Science and Technology (CREST) program from the Japan Science and Technology (JST) Corporation (to Y.K.). We wish to thank M. Kimura for assistance with the analysis and $\mathrm{T}$. Yoshiike for helpful discussions.

\section{Author contributions}

Conceived and designed the experiments: MH. Supervised the study: KK. Performed the experiments: MH YO NE. Analyzed the data: MH YK. Wrote the manuscript: MH KK.

\section{Additional information}

Supplementary information accompanies this paper at http://www.nature.com/ scientificreports

Competing financial interests: The authors declare no competing financial interests. License: This work is licensed under a Creative Commons Attribution-NonCommercial-ShareAlike 3.0 Unported License. To view a copy of this license, visit http://creativecommons.org/licenses/by-nc-sa/3.0/

How to cite this article: Honma, M., Endo, N., Osada, Y., Kim, Y. \& Kuriyama, K. Disturbances in equilibrium function after major earthquake. Sci. Rep. 2, 749; DOI:10.1038/ srep00749 (2012). 